\title{
Hygroscopic Materials and Characterization Techniques for Fiber Sensing Applications: A Review
}

\author{
Muhammad Arif Riza, ${ }^{1}$ Yun Ii Go, ${ }^{1 *}$ Robert R. J. Maier, ${ }^{2}$ \\ Sulaiman Wadi Harun, ${ }^{3}$ and Siti Barirah Anas ${ }^{4}$ \\ ${ }^{1}$ School of Engineering and Physical Sciences, Heriot-Watt University Malaysia, \\ No 1, Jalan Venna P5/2, Precinct 5, Putrajaya 62200, Malaysia \\ ${ }^{2}$ Institute of Photonics and Quantum Sciences, Heriot-Watt University, \\ Campus The Avenue, Edinburgh EH14 4AS, United Kingdom \\ ${ }^{3}$ Department of Electrical Engineering, Faculty of Engineering, University of Malaya, \\ Kuala Lumpur 50603, Malaysia \\ ${ }^{4}$ Wireless and Photonics Networks Research Centre, Universiti Putra Malaysia, \\ Selangor 43400, Malaysia
}

(Received June 26, 2020; accepted October 8, 2020)

Keywords: adsorption, characterization, deliquescence, material, hygroscopicity

Various characterization techniques are used in the study of materials in order to understand their properties. Data and observation recording characterization of a material is done by researchers for various properties within various environments. One of the many properties that make a material attractive for use in certain devices is hygroscopicity. Various techniques for characterizing hygroscopicity have been developed and utilized. However, since these techniques are not usually the highlights of studies, there has been limited acknowledgement of the usefulness of hygroscopicity testing. This review introduces several established characterization techniques allowing researchers to fully characterize materials in terms of their hygroscopicity. The full potential of hygroscopic materials can be seen in their use in various fiber sensors. The deformation of materials upon exposure to water molecules can be utilized to perturb sensing elements in fiber sensors, allowing the user to take full advantage of the hygroscopicity of the material.

\section{Introduction}

Material science throughout the years has covered various properties of materials including physical and chemical properties. Materials that are capable of absorbing, adsorbing, and desorbing water molecules while undergoing slight deformation of their nanostructure are considered to be hygroscopic materials. Hygroscopicity, a physical property of a material, is a measure of how well the material can absorb and release water molecules. Certain materials may expand or deform upon exposure to water molecules. ${ }^{(1)}$ This property can enable a material to be used in an environmental sensor or environmentally activated actuator. However, the hygroscopicity of materials has often had little discussion in studies since it 
is often overshadowed by the main objective of the studies. For example, a paper reporting the development of a humidity/chemical sensor will have its results and discussion weighted heavily on the sensor performance rather than the hygroscopic characterization of the material, despite it being part of the actual sensing mechanism and behavior of the humidity sensor itself. Therefore, this review brings together various studies that focus on techniques for characterizing hygroscopic materials for environmental sensing applications.

\subsection{Water absorption and desorption in materials}

Hygroscopic materials behave similarly to a sponge upon exposure to water molecules in the environment. This behavior can be modelled via Fick's law of diffusion: ${ }^{(2)}$

$$
F_{x}=-D\left(\frac{\partial c}{\partial t}\right)
$$

where $F_{x}$ is the flux, $D$ is the diffusion coefficient, and $\partial c / \partial t$ is the concentration change of the material with unit of time. However, this model only applies to steady-state diffusion where the concentration of molecules does not vary in time. In the case of changing environmental humidity, the concentration of water molecules in air changes. Therefore, another form of Fick's law is introduced:

$$
\frac{\partial c}{\partial t}=D\left(\frac{\partial^{2} c}{\partial x^{2}}+\frac{\partial^{2} c}{\partial y^{2}}+\frac{\partial^{2} c}{\partial z^{2}}\right) .
$$

With the involvement of each plane of dimensions at a point in the Cartesian plane, Eq. (2) describes the increment or decrement of the amount of diffused molecule on a plane with time. For a study involving the diffusion of water molecules within a thin film sample on a two-dimensional plane, we have

$$
\frac{\partial c}{\partial t}=D\left(\frac{\partial^{2} c}{\partial x^{2}}\right)
$$

To derive a version of Eq. (3) that involves the mass of the sample, the mass adsorbed by the material at equilibrium and the mass adsorbed at time $T$ are taken into account to obtain the following equations:

$$
\frac{M_{T}}{M_{E}}=1-\sum_{n=0}^{\infty} 8 \exp \left(\frac{-\frac{D(2 n+1)^{2} \pi^{2} t}{4 L^{2}}}{(2 n+1)^{2} \pi^{2}}\right),
$$




$$
\frac{M_{T}}{M_{E}}=\frac{4\left(\frac{D t}{\pi}\right)^{\frac{1}{2}}}{L} .
$$

Equation (5) is a simplified version of Eq. 4 that relates the adsorbed mass ratio and the sample thickness, as suggested in Refs. 2 and 3. By incorporating the original Fick's law of diffusion into a simpler model, the hygroscopicity of certain materials can be characterized. Furthermore, should more detailed, three-dimensional modeling of hygroscopicity that takes into account the $x, y$, and $z$ directions be required, Eq. (2) can be expanded accordingly.

\subsection{Effect of nanostructure on hygroscopic performance}

In addition to the thickness and dimensions of a material, its nanostructure plays a role in determining hygroscopic performance. A structure with a porous nature tends to possess greater hygroscopicity. This is due to the pores acting as sites for the diffusion of molecules within the material itself. ${ }^{(3)}$ Furthermore, porous nanostructures offer increased surface area compared with smooth and impermeable nanostructures. Additionally, nanostructures with high roughness will always have a large surface area and allow the easier adsorption of water molecules. Therefore, the characterization of material hygroscopicity should at least involve some form of microscopic observation of the nanostructure to also obtain details of its roughness, which is based on the average number of peaks and troughs present on the nanostructure.

\section{Hygroscopic Materials}

A wide variety of materials can behave hygroscopically provided they have sufficient surface area or porosity. Some studies have reported an increase in the mass of hygroscopic materials with the uptake of water while others have reported changes on the surface of the materials. Therefore, the hygroscopic behaviour may vary among materials in terms of mass of water uptake or nanostructure form. Figure 1 shows various forms of hygroscopic nanostructures.

\subsection{Soluble materials}

Soluble materials are usually extremely hygroscopic and can be considered as deliquescent materials. Deliquescence is a behavior wherein a solid absorbs an extremely large amount of water, resulting in its transformation into a liquid form. ${ }^{(10)}$ An example of a material that exhibits this behavior is sodium chloride $(\mathrm{NaCl})$. Crystalline $\mathrm{NaCl}$ will start to exhibit deliquescent behavior upon being exposed to its deliquescence relative humidity (DRH). In other words, the $\mathrm{NaCl}$ will become diluted and experience a phase change from solid crystals to an aqueous liquid phase upon exposure to relative humidity $(\mathrm{RH})$ that exceeds the DRH. It was also noted that $\mathrm{NaCl}$ particles with a smaller diameter have lower DRH. As a result, 


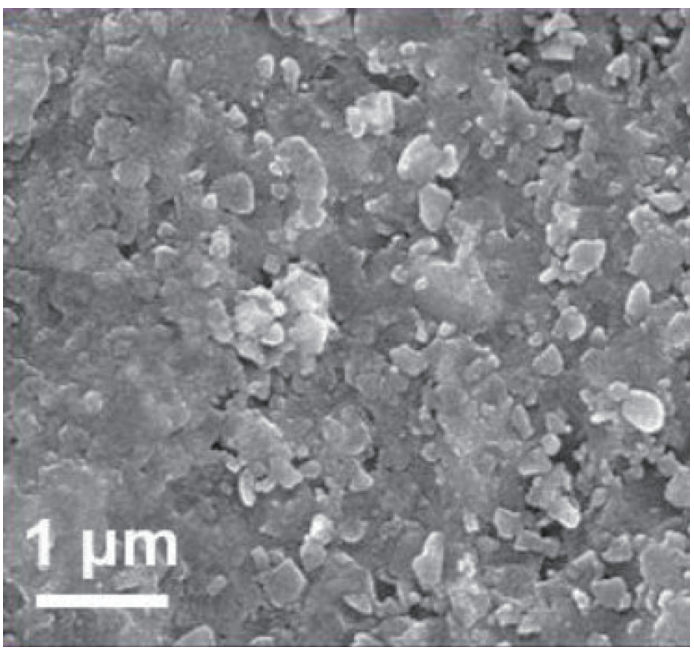

(a)

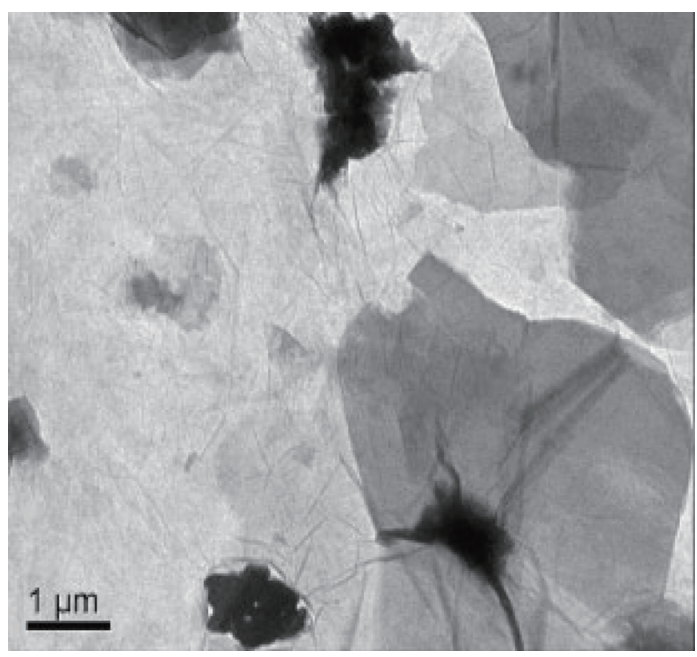

(c)

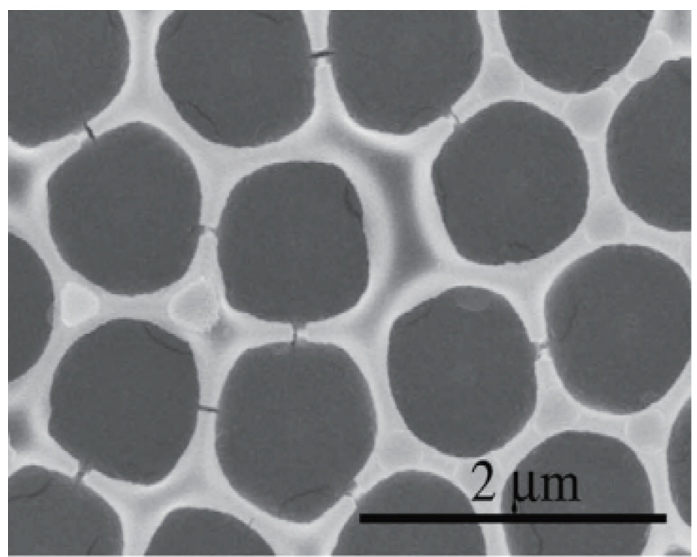

(e)

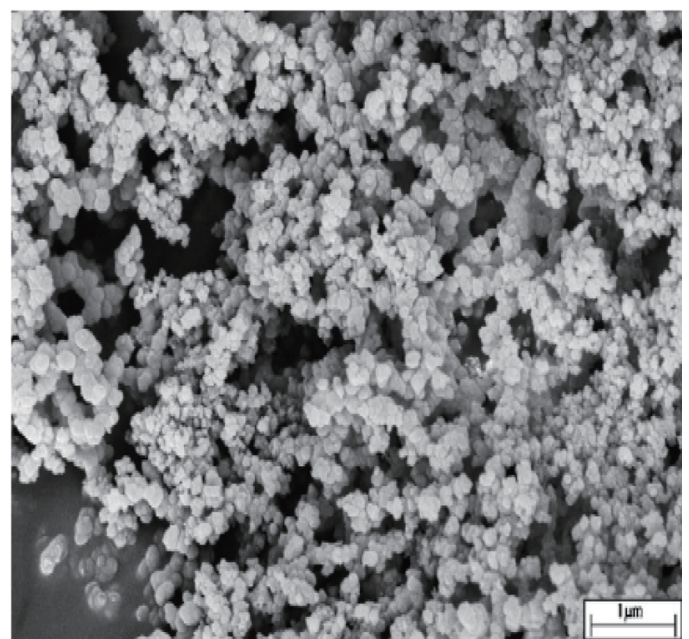

(b)

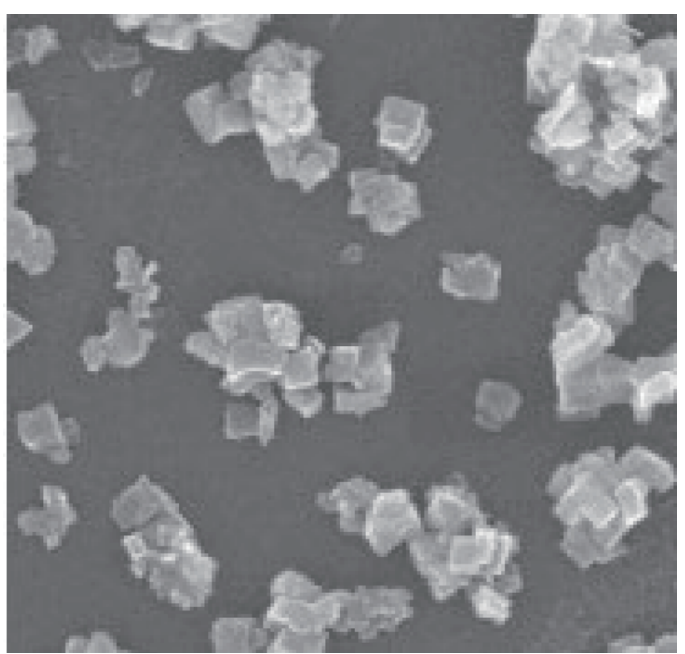

(d)

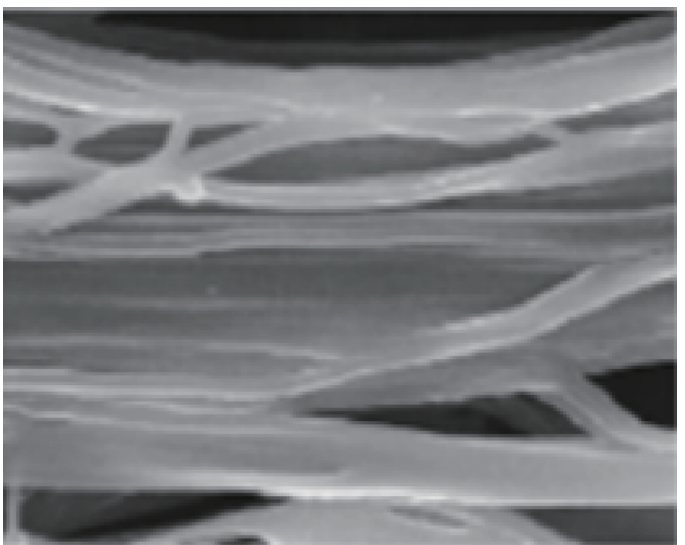

(f)

Fig. 1. Nanostructures of different materials that can exhibit hygroscopic behavior: (a) $\mathrm{BaTiO}_{3},{ }^{(4)}$ (b) aluminum hydroxide, ${ }^{(5)}$ (c) graphene oxide, ${ }^{(6)}$ (d) indium oxide $\left(\mathrm{In}_{2} \mathrm{O}_{3}\right),{ }^{(7)}$ (e) $\mathrm{TiO}_{2}$ film, ${ }^{(8)}$ and (f) polyimide nanofibers. ${ }^{(9)}$ 
small-diameter $\mathrm{NaCl}$ crystals will be more prone to changing into a liquid form at lower RH. ${ }^{(11)}$ Deliquescent materials usually must be stored appropriately for preservation and their implementation.

\subsection{Polymers}

\subsubsection{Polydimethylsiloxane (PDMS)}

As a composite of a polymer and a silicone-based material, PDMS is widely used in many different devices. Its stretchability and low Young's modulus allow it to be embedded in soft surfaces such as textiles. The material exhibits high permeability to water vapor but low permeability to liquid water and large water droplets. A study ${ }^{(12)}$ revealed that materials that have many micropores tend to have a low Young's modulus, which renders them flexible. These advantageous properties allow PDMS to be implemented in capacitive humidity sensors embedded in soft textiles.

\subsubsection{Polyimide}

Another known hygroscopic polymer is polyimide. This material is well known for its reversible expansion properties upon water discharge from its nanostructure. This makes it a reliable sensor coating for sensor mechanisms that rely on mechanical stress. An example of the use of polyimide can be seen in Ref. 14, where polyimide is coated on a fiber Bragg grating (FBG)-based sensor for humidity sensing. Upon exposure to humidity, the polyimide coating expands and induces longitudinal strain on the FBG segment of the fiber. This perturbs the core gratings and produces a signal that can be translated into reliable data. The signal is in the form of wavelength shifts in the FBG and the polyimide coating facilitates the expansion or deformation of the FBG upon water uptake, as shown in Fig. 2. The study also reported good
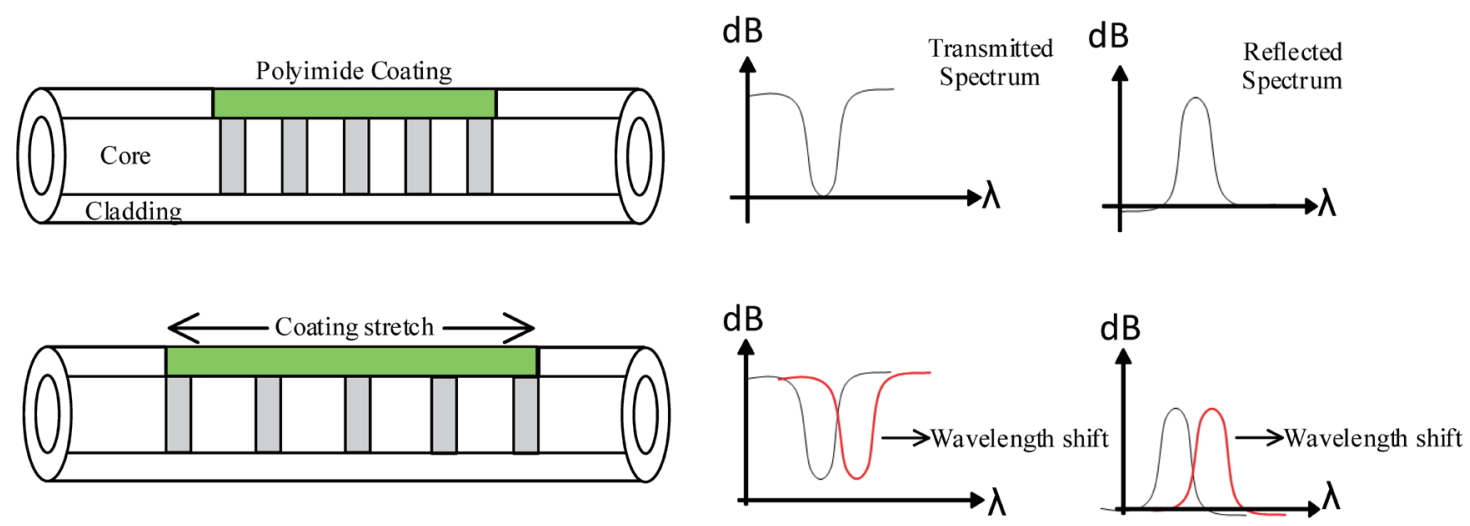

Fig. 2. (Color online) Polyimide expansion on an FBG sensor. 
reliability of the polyimide-coated FBG sensor since it showed linear changes when exposed to increasing and decreasing humidity.

\subsubsection{Polyethylene glycol (PEG)}

Polymeric glycols are another example of porous polymers that show hygroscopic behavior. Their porous nature allows water molecules to easily absorb within their structure. Lee and Park ${ }^{(13)}$ reported on the combination of an $\mathrm{O}_{2}$-sensitive dye polymer matrix and PEG to form a humidity sensor. When the PEG ratio within the polymer complex was increased, more pores were found to exist within the thin-film structure, which enhanced the water retention of the material.

\subsection{Metal oxides}

\subsubsection{Zinc oxide}

As one of the more abundant metal sources, zinc oxide boasts various properties that are of interest for a wide variety of applications in various fields. Zinc oxide possesses semiconductive properties as well as hygroscopic properties, depending on its nanostructure. Various synthesis routes, such as sol-gel, hydrothermal, and solid-state reactions, have been established for zinc oxide. Nowadays, new and modified synthesis methods to produce zinc oxide with various nanostructures are being explored. Certain parameters in the synthesis process, such as the annealing temperature, $\mathrm{pH}$ level, precursor salts, and additives, can influence the nanostructure of the obtained zinc oxide. ${ }^{(14,15)}$

Zinc oxide has been applied as a humidity sensor that exploits its hygroscopic behavior. By coating an FBG-based optical sensor with $\mathrm{ZnO}$, detection of relative humidity becomes possible. This has been explored by Aris, ${ }^{(16)}$ who employed a nanostructure of hybrid flakes of zinc oxide as a sensor layer on an FBG. They compared a bare FBG with a ZnO-sensitized FBG for the detection of relative humidity in the range of 55-80\%. The ZnO-sensitized FBG showed wavelength shifts that were noticeably greater than those of the bare FBG. This indicates that the $\mathrm{ZnO}$ coating enhances the humidity sensitivity for FBG sensors. Since $\mathrm{ZnO}$ can be easily doped and modified, it should be possible to further improve the properties of $\mathrm{ZnO}$ nanostructures. In theory, if a $\mathrm{ZnO}$ nanostructure is made with higher roughness that provides a greater number of adsorption sites for water molecules, its hygroscopicity will be enhanced. This could lead to a sensor with higher sensitivity. Figure 3 shows different nanostructures obtained in other studies.

\subsubsection{Lanthanum oxide}

Lanthanum oxide $\left(\mathrm{La}_{2} \mathrm{O}_{3}\right)$ is a rare-earth material that possesses interesting electrochemical properties. This material is capable of exhibiting partially hygroscopic properties upon exposure to humidity. According to Gangwar et al., ${ }^{(21)} \mathrm{La}_{2} \mathrm{O}_{3}$ reacts hygroscopically upon the adsorption of water molecules onto molecular bonds before the hydroxylation reaction 


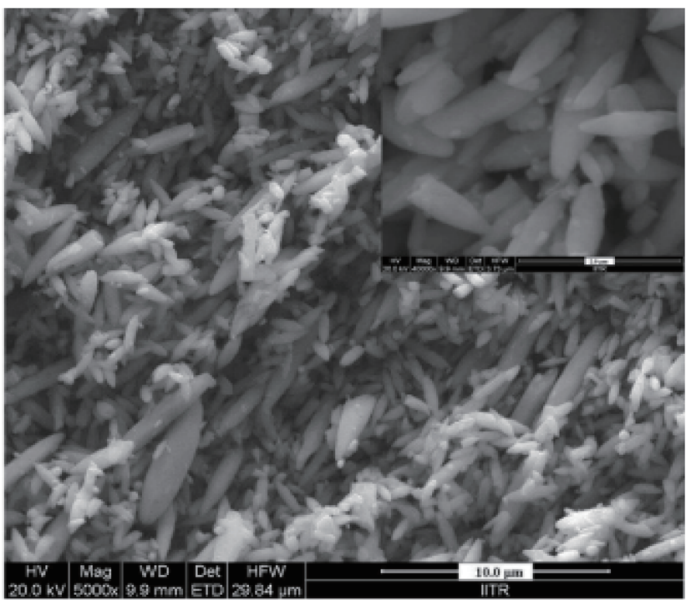

(a)

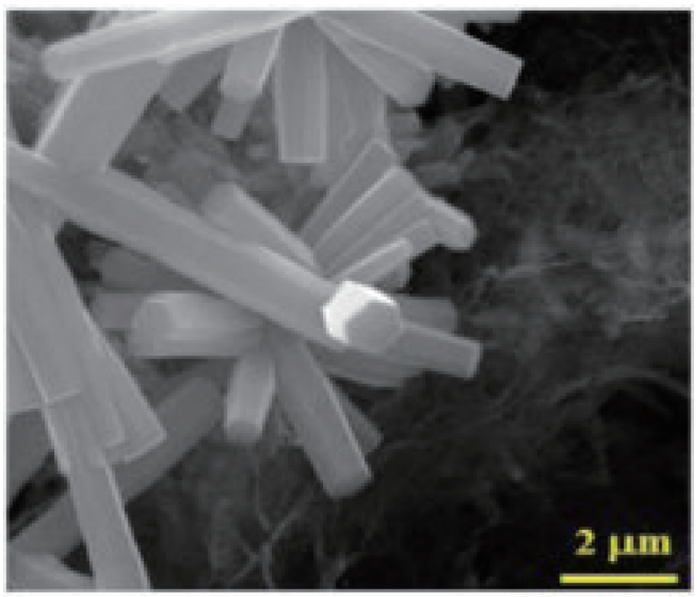

(c)

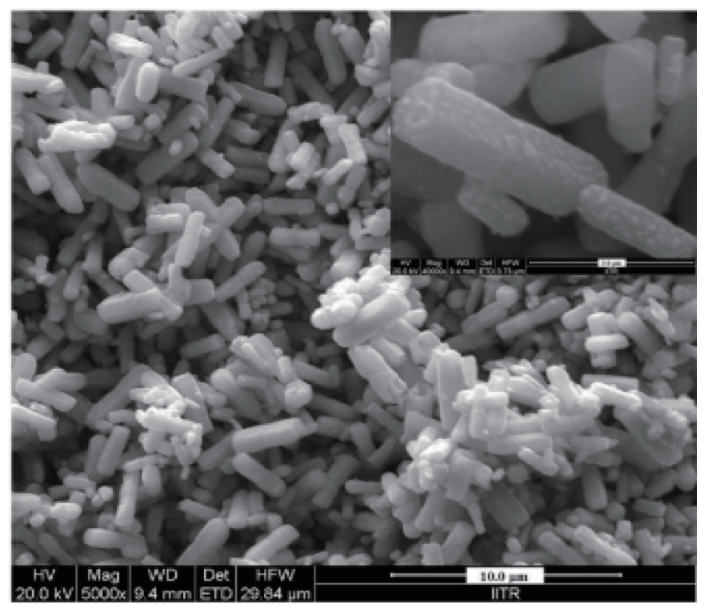

(b)

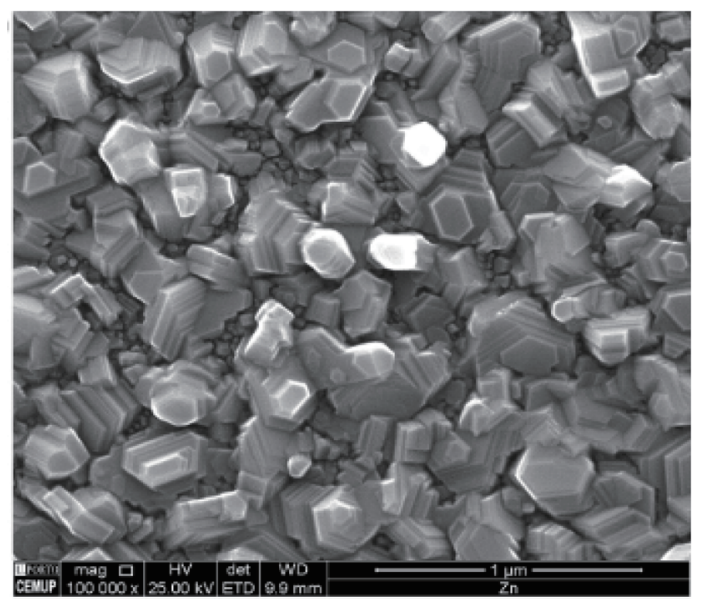

(d)

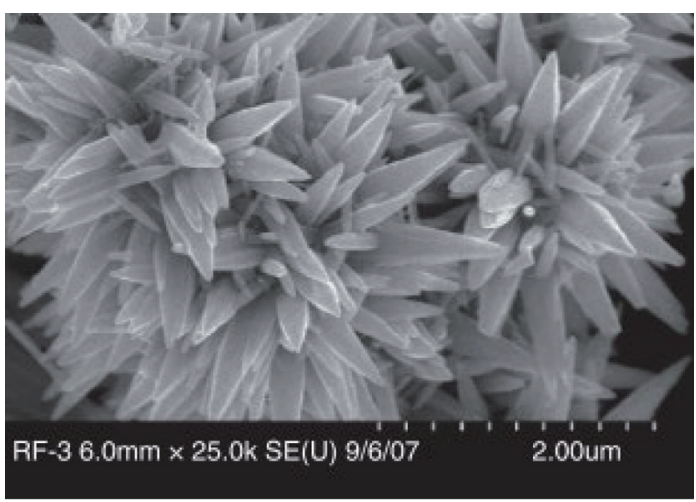

(e)

Fig. 3. (Color online) Zinc oxide nanostructures obtained by various synthesis methods: (a) zinc oxide grains obtained from zinc chloride precursor sol-gel, ${ }^{(17)}$ (b) short hexagonal zinc oxide rods obtained from zinc acetate precursor sol-gel, ${ }^{(17)}$ (c) hexamethylenetetramine (HMTA)-enhanced zinc oxide nanoflakes obtained by hydrothermal method, ${ }^{(18)}$ (d) zinc oxide crystal islands directly deposited via e-beam deposition from zinc metal precursor (no synthesis route), ${ }^{(19)}$ and (e) zinc oxide microflowers obtained through wet-chemical-based synthesis with $\mathrm{NaOH} .{ }^{(20)}$ 
begins. $\mathrm{La}_{2} \mathrm{O}_{3}$ and water molecules undergo a hydroxylation reaction that alters the molecular arrangement of $\mathrm{La}_{2} \mathrm{O}_{3}$. This absorption causes $\mathrm{La}_{2} \mathrm{O}_{3}$ to be reduced to $\mathrm{La}(\mathrm{OH})_{3}$, which has slightly different properties from its precursor. Being a metal oxide, the hygroscopic behavior of $\mathrm{La}_{2} \mathrm{O}_{3}$ can be enhanced by doping other metal elements, as reported elsewhere. ${ }^{(21)}$ This material can be implemented in fiber sensors provided that the $\mathrm{La}(\mathrm{OH})_{3}$ can undergo a reversible reaction back to its original molecule $\left(\mathrm{La}_{2} \mathrm{O}_{3}\right)$ or the hydroxylation reaction upon the uptake of water by $\mathrm{La}_{2} \mathrm{O}_{3}$ can be prevented from occurring.

\subsubsection{Copper oxide powder}

Copper oxide synthesized by the sol-gel method from a salt-based precursor has been found to exhibit a slightly hygroscopic behavior. ${ }^{(22)}$ In the completely dry state, copper oxide does not exhibit noticeable water adsorption or absorption. However, in the presence of nearby volatile compounds, it develops hygroscopic properties. Volatile compounds, such as acetic acid and formaldehyde, tend to supply hydroxyl groups that adhere to the surface of the copper oxide. These hydroxyl groups are hydrophilic and aid the adsorption of water molecules via hydrogen bonding. Without the presence of volatile compounds, copper oxide only shows hygroscopic properties when exposed to a very high relative humidity of $99 \%$. At such a high humidity, the material is considered to be completely hydrated. A logical explanation for the low hygroscopicity of copper oxide at normal humidity is that its roughness is low, as observed by AFM investigation in the same study. ${ }^{(23)}$ This low roughness indicates a smooth surface of the material, which makes it difficult for water molecules to adsorb onto the surface.

\subsection{Other materials}

\subsubsection{Wood composites}

As a basis of most components used in building, wood composites are used in conjunction with polymers and cements. The hygroscopic property of wood allows the humidity of a room to be regulated. Because of the unique nanostructure of wood, water uptake causes its expansion, and wood can show a notable difference in mass between its dry and wet states. A room whose surfaces are made of pure cement shows much higher humidity than one whose surfaces are made of a composite of cement with wood layers within it. This shows that the excellent hygroscopic properties of wood allow it to reduce the humidity in an enclosed space. ${ }^{(24)}$ However, it may not be possible to integrate wood in certain smaller materials or devices, such as optical interferometry sensors, as its refractive index cannot be changed. In addition, it may not be cost-effective to produce nanostructures of wood.

\subsubsection{Ceramic materials}

Ceramic materials are often made of more than one different metal source with an atom arrangement of $\mathrm{ABX} 3$. As an example, barium titanate $\left(\mathrm{BaTiO}_{3}\right)$ is capable of water intake and retention owing to its extremely porous structure. ${ }^{(4)}$ Using this property, Liang et al. ${ }^{(4)}$ 
attempted to apply $\mathrm{BaTiO}_{3}$ as a capacitive humidity sensor by employing an aerosol deposition technique to deposit the ceramic material to various thicknesses between 0.1 and $10 \mu \mathrm{m}$. The $\mathrm{BaTiO}_{3}$-based sensor exhibited its greatest humidity-sensing performance at a $\mathrm{BaTiO}_{3}$ thickness of $3 \mu \mathrm{m}$ as a result of the material having a high RMS roughness and particle size. However, the sensitivity began to decrease above a thickness of $6.0 \mu \mathrm{m}$. According to the results of that study, as the thickness of the sensor material increases, the hydrophilicity and pore volume increase but the open pore ratio decreases. The sensitivity of the sensor element is determined by a combination of the hydrophilicity, pore volume, and open pore ratio. Optimization of the three parameters may lead to improved sensitivity for capacitive-based sensors.

\subsubsection{Agar gel}

Some materials in the form of a gel can also adopt hygroscopic properties. Agar gel has been implemented in sensors that rely on the deformation of the sensing element. This is because it is a polysaccharide complex that deforms upon exposure to water molecules. Caponero et al. ${ }^{(25)}$ attempted to coat agar gel as the main sensing element onto an FBG-based humidity sensor in order to monitor humidity levels in cultural heritage stones. Upon water uptake, the agar gel deformed and induced strain on the Bragg gratings. This induced a wavelength shift that was translated into data on the relative humidity. While it is easy to use agar gel as a sensitized coating for sensing, it should be noted that this material is not thermally robust. At temperatures above $85^{\circ} \mathrm{C}$, it melts, making it unsuitable for monitoring humidity in high-temperature environments. ${ }^{(23)}$

\subsubsection{Graphene}

A longer-known advanced material that is a derivative of countless possible arrangements of carbon atoms is graphene. A sheet arrangement of graphene oxide (GO) monolayers, shown in Fig. 4, possesses various oxygen functional groups that are hydrophilic, thus providing the

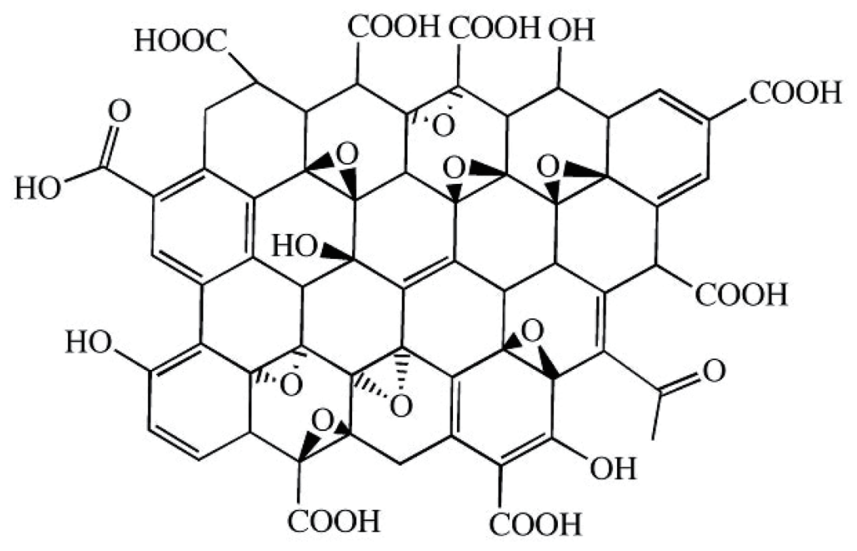

Fig. 4. Graphene structure. ${ }^{(26)}$ 
nanostructure with good hygroscopicity for sensing applications. Perera et al. ${ }^{(26)}$ examined the hygroscopic behavior of GOs using a quartz crystal microbalance (QCM). They demonstrated the adsorption and water uptake of GOs upon exposure to humidity. They reported that sheet nanostructured GOs exhibit two separate adsorption behaviors depending on the humidity. At low RH, adsorption on the surface edges and functional groups is the predominant behavior. At a higher RH of approximately $56 \%$, the water molecules are absorbed into the interstitial layers within the carbon sheet. The latter absorption process is the dominant phenomenon at higher RH\%. ${ }^{(26)}$ These two different RH-dependent water uptake phenomena may pose a challenge in designing humidity sensors for monitoring a wide range of $\mathrm{RH} \%$. A suitable additive or modifier compound could be added to prevent GO from switching between the two hygroscopic modes.

To summarize this section, hygroscopic materials with various nanostructures have been implemented in a range of applications. Table 1 shows the materials, their structural forms, and their potential applications.

\section{Hygroscopicity Characterization of Materials}

Various techniques have been applied to measure the hygroscopicity of materials to obtain a greater understanding of their behavior and properties. Some techniques require improvisation before they can be applied in hygroscopic characterization. In this section, we discuss some of the studies that have involved hygroscopic characterization techniques.

\subsection{Microscopy characterization}

Microscopic observation is a staple component of many studies, especially in material science. Through microscopic images, the hygroscopicity of a material can also be observed. A material may deform or show a certain misconfiguration in its nanostructure upon exposure to humidity. A qualitative analysis can be turned into a quantitative analysis through the

Table 1

Various hygroscopic materials and their nanostructures.

\begin{tabular}{lcccc}
\hline Material & Structural form & Porosity/surface area & Applications & Ref \\
\hline Zinc oxide & Needle/cylinder & $\begin{array}{c}\text { High depending on size of } \\
\text { needles }\end{array}$ & Various & $(17)$ \\
\hline $\begin{array}{l}\text { Lanthanum oxide } \\
\begin{array}{l}\text { Barium titanate } \\
\left(\mathrm{BaTiO}_{3}\right)\end{array}\end{array}$ & Sheet & $\begin{array}{c}\text { Medium but may change if } \\
\text { converted to } \mathrm{La}(\mathrm{OH})_{3}\end{array}$ & Sensors, capacitors, dopant & $(21)$ \\
\hline $\begin{array}{l}\text { Polyimide } \\
\mathrm{CaCl}_{2}\end{array}$ & Polymer & Very high & Capacitive humidity sensors & $(4)$ \\
\hline PDMS & Flexible gel polymer & High & FBG-based humidity sensors & $(27)$ \\
\hline Agar & Gel polysaccharides & High & Capacitive, flexible humidity sensors & $(12)$ \\
\hline Graphene & Monolayer sheet & $\begin{array}{c}\text { Variable depending on carbon } \\
\text { arrangement }\end{array}$ & Sensors & $(23)$ \\
\hline
\end{tabular}


measurement of such deformation. As an example, Kim et al. ${ }^{(28)}$ analyzed the hygroscopicity of the perovskite $\mathrm{APbI}_{3} / \mathrm{PEO}$ through its deformation upon the introduction of humidity, which manifested as black spots appearing on the nanostructure surface. The area of black spots was measured with a graphic tool and compared with the area of white spots on a selected part of the sample. The ratio of black spots to white spots was used as the data output for characterization. This technique may vary with the type of nanostructure since deformation behavior can vary among materials.

\subsection{Tandem nano-differential mobility analysis (TDMA)}

TDMA reveals the hygroscopic properties of a material through a setup involving various components. Figure 5 shows the arrangement employed by MacMillan et al. ${ }^{(30)}$ to obtain the hygroscopic properties of imidazolium aerosols. The basic principle of this complex system is a continuous flow of an aerosol or gas of the subject material from one chamber with a set dry humidity $(0 \% \mathrm{RH})$ to a chamber with a different set humidity. Normally, the growth factor

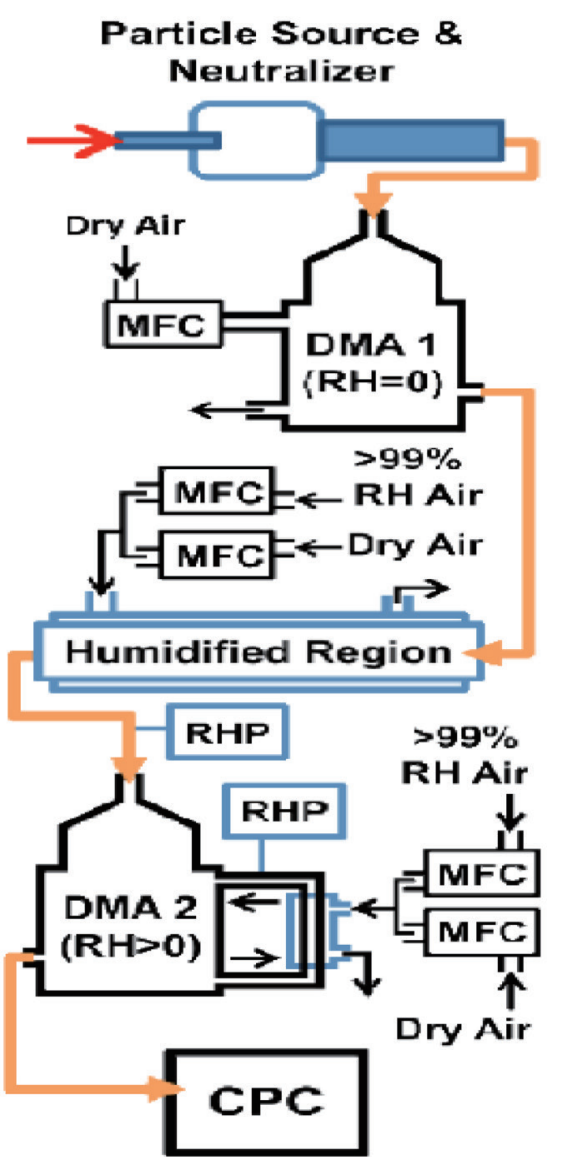

Fig. 5. (Color online) System for TDMA. ${ }^{(6)}$ 
is one form of data that can be provided by TDMA. By comparing the ratio of the particle diameters pre- and post-humidity exposure, growth factor curves can be obtained. A higher growth factor corresponds to greater water uptake by a material. This technique can be used to analyze materials that are in the form of aerosols. However, in addition to the complex setup, extra measures must be taken to nebulize samples into aerosol forms with known diameters.

\subsection{Sum frequency generation spectroscopy (SFG)}

SFG is used to gain a molecular understanding of hydrophobicity in materials. SFG employs a Nd:YAG picosecond laser pump with an optical parametric generator system. As depicted in Fig. 6, infrared (IR) light, $\omega_{I R}$, and visible light, $\omega_{V I S}$, sources of the laser pump are directed to the sample and a detector picks up the reflected ray, which is the sum of $\omega_{I R}$ and $\omega_{V I S}$, to analyze the state of the bonds in the sample. It bears a close resemblance to X-ray photoelectron spectroscopy except for the source of the laser. While it does not give direct data on the hygroscopicity of a material, it can show specific molecular bonds within a compound. Chemical bonds tend to experience molecular strain upon the adsorption of water molecules at the surface. Such strain can be reflected via the spectra generated from the SFG system. This allows the detection of water molecules adsorbing onto the surface of a material. ${ }^{(29)}$

\subsection{QCM}

A QCM is a highly sensitive weight balance that can be used for the determination of the microscopic adsorption of water molecules on a material. Its operations rely on a quartz crystal disc and the piezoelectric effects of electrodes connected to the disc. The electrodes are attached to the disc so that the disc is sandwiched between the electrodes. The voltage applied to the electrodes causes the quartz crystal disc to undergo microbending. After halting the voltage supply, the quartz disc resonates at a resonant frequency until all the energy in

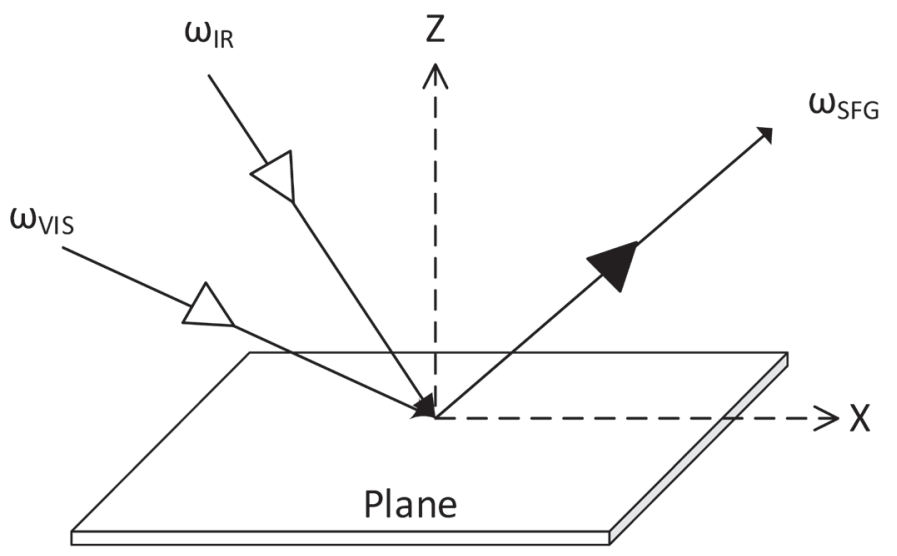

Fig. 6. SFG theory. 
the disc has dissipated. ${ }^{(30)}$ The resonance frequency is derived via a frequency shift equation proposed by Sauerbrey ${ }^{(31)}$ in 1959 relating the frequency shift and the mass detected by the disc. According to the equation, the frequency shift is proportional to the mass of the sample. Characterizing a sample by this technique requires the deposition of a very thin film of the sample on the crystal disc before the voltage is supplied. For instance, Chiarello et al. ${ }^{(32)}$ deposited samples on cleaned quartz discs by vapor deposition before applying a voltage. A recent study involved the coating of graphene-based materials and the direct application of the whole system comprising the graphene film and a QCM as a humidity sensor with the graphene as the main sensing element. ${ }^{(6)}$ From the results of those studies, it can be concluded that the use of a QCM for hygroscopic characterization can provide highly accurate water adsorption readings in the form of mass changes. However, preparing and attaching a sample onto the quartz disc requires a suitable thin-film deposition method. Furthermore, the investigation may be prone to errors since additional steps are required, especially when comparing two or more different materials for which separate deposition on different quartz discs is necessary.

\subsection{Moire interferometry}

Originally used in the electronics industry to test for thermally induced deformation on electrical component packages, Moire interferometry was adapted for hygroscopic characterization by Stellrecht et al. ${ }^{(33)}$ Moire interferometry, as shown in Fig. 7, is an optical

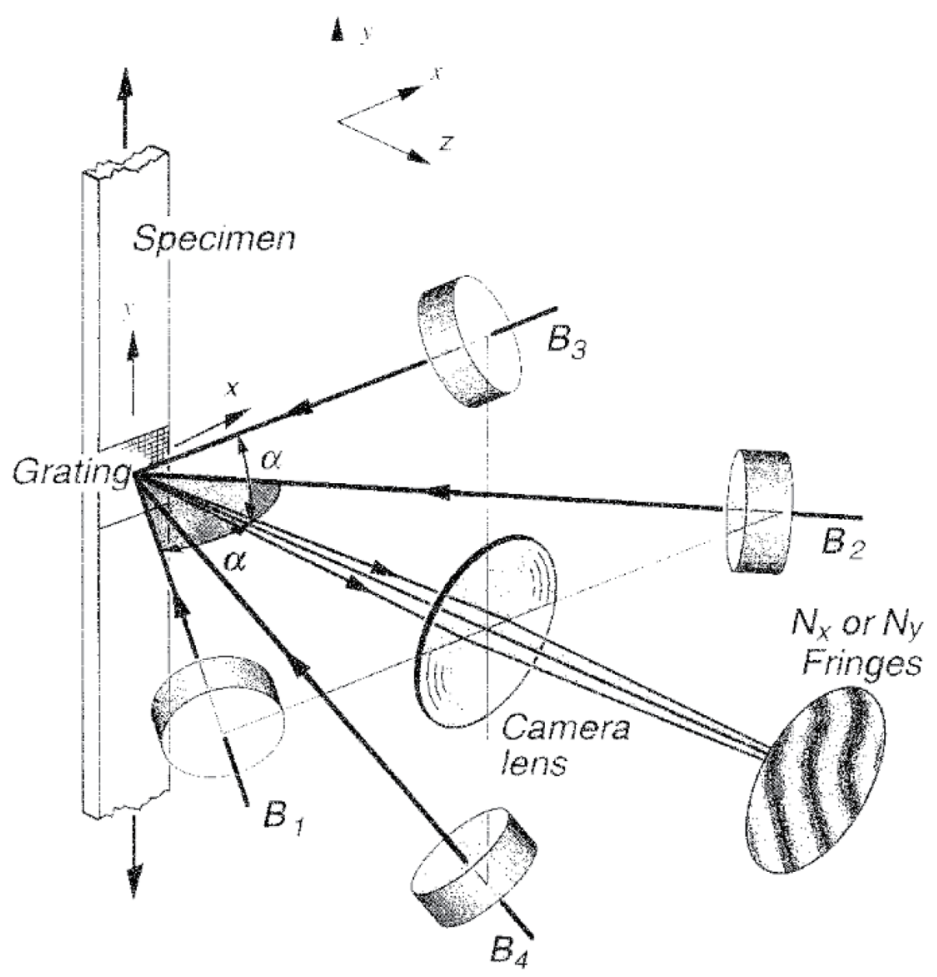

Fig. 7. Moire interferometry as depicted by Han et al. ${ }^{(34)}$ 
real-time whole-field displacement measurement technique that can measure and present in-plane deformation data accurately. The data are in the form of contour maps of the displacement field appearing on the measured subject material. ${ }^{(34)}$ This technique involves multiple optical beams that are directed to the subject material at different angles. The diffraction of the beams owing to deformed gratings or fringes on the subject material results in the formation of diffracted beams. These diffracted beams, when interloped with the incident beams, create constructive and destructive interference called Moire patterns. The subject material is usually in the form of 2-mm-thick solid slabs of a polymeric material. In the work by Stellrecht et al., ${ }^{(33)}$ a chamber was employed in which the relative humidity was manipulated. Owing to the real-time observation capability of this technique, the deformation of a material as a result of exposure to humidity can be determined. However, the technique performs best with materials that form fringes after baking. Such materials with surfaces capable of holding fringes are usually plastic-encapsulated microplastics. This could limit the use of this technique on other types of materials.

\subsection{Weighing method}

When nanostructures adsorb or absorb water molecules, their weight tends to slightly increase. This can be used as a measurement method for characterizing the hygroscopicity of different materials. Zheng et al. ${ }^{(3)}$ applied this technique to quantify the hygroscopicity of two different materials. The materials were spread on a glass slide and weighed before and after exposure to a set relative humidity within an enclosed chamber. The data of weight gained was plotted against the duration of humidity exposure, where the humidity was increased every $3 \mathrm{~h}$. This method allows one to quantitatively characterize the hygroscopic capability of a sample from its weight change. Although the technique is flexible and can characterize most materials in a solid or thin-film form, it may also require microscopic observation to fully characterize and understand the mode of the nanostructural expansion or water-holding mechanism.

\subsection{Advantages and limitations of hygroscopic characterization techniques}

Hygroscopic analysis and characterization techniques vary greatly. Every technique has its advantages and limitations, such as the requirement of specialized equipment and the mode of data collection. Some of these techniques can be combined to produce more reliable results. Table 2 presents the advantages and limitations of each technique.

From the literature on various hygroscopic characterization techniques, it can be clearly seen that each technique has its pros and cons. In order to examine hygroscopic characterization as completely as possible, more than one characterization technique can be used. Each technique may supplement data obtained by another technique, giving a more accurate depiction of the hygroscopic behavior of each material. The decision to select a characterization technique must also be based on the form of the subject material and its expected application. 
Table 2

Advantages and limitations of each testing technique.

\begin{tabular}{|c|c|c|}
\hline Technique & Advantages & Limitations \\
\hline Microscopy & $\begin{array}{l}\text { - Provides qualitative data on morphology of } \\
\text { nanostructure } \\
\text { - Possible with both thin films and powder } \\
\text { samples }\end{array}$ & $\begin{array}{l}\text { - Must be combined with another technique } \\
\text { for greater accuracy of data } \\
\text { - Measurement of pore size may not be } \\
\text { completely accurate if measuring software } \\
\text { is used }\end{array}$ \\
\hline TDMA & $\begin{array}{l}\text { - Can analyze materials in fine aerosol forms } \\
\text { and gaseous forms. } \\
\text { - One of the more accurate and reliable } \\
\text { techniques for providing growth factor of } \\
\text { materials with adsorbed water }\end{array}$ & $\begin{array}{l}\text { - Intricate assembly of system } \\
\text { - Costly } \\
\text { - Diameter of sample must be set/known, } \\
\text { requiring another analysis technique }\end{array}$ \\
\hline SFG & $\begin{array}{l}\text { - Provides molecular bond information in } \\
\text { response to humidity }\end{array}$ & $\begin{array}{l}\text { - Requires specialized equipment for beam } \\
\text { propagation }\end{array}$ \\
\hline QCM & $\begin{array}{l}\text { - More accurate quantitative result than } \\
\text { weighing method }\end{array}$ & $\begin{array}{l}\text { - Samples must be well deposited as thin } \\
\text { film on the quartz slab before initiating } \\
\text { measurements }\end{array}$ \\
\hline Moire interferometry & $\begin{array}{l}\text { Established and reliable method for testing } \\
\text { hygroscopic properties or moisture } \text { induced } \\
\text { deformation for PEMs }\end{array}$ & $\begin{array}{l}\text { - Samples must be moulded into a specific } \\
\text { form with fringes on its surface }\end{array}$ \\
\hline Weighing method & $\begin{array}{l}\text { - Accessible and easy preparation } \\
\text { - Suitable for more than one form of sample } \\
\text { - Does not require special shape or form of } \\
\text { sample }\end{array}$ & $\begin{array}{l}\text { - Depending on the accuracy and } \\
\text { reliability of the weight balance, multiple } \\
\text { measurements may be required to ensure } \\
\text { acceptable error margin }\end{array}$ \\
\hline
\end{tabular}

\section{Integration of Hygroscopic Materials in Sensing Enhancement}

The ability of a material to exhibit deformation upon exposure to water molecules enables its use as an environmental sensor. This allows monitoring of the relative humidity of an environment. Fiber sensors can be used in conjunction with hygroscopic materials for humidity sensing. Hygroscopic materials used in optical-based sensors are mainly used for the detection of humidity and chemicals. Optical-based sensors include interferometers, which take advantage of the change in the refractive index of the sensing material in the detection of environmental stimuli. A family of optical sensors that takes full advantage of hygroscopic materials are FBG sensors. As mentioned in previous sections, the expansion and contraction of hygroscopic materials affect the perturbation of light traveling within the core of grated fibers. This induces a shift in the spectral wavelength, which is interpreted via external computing to represent detection data. Table 3 shows optical sensors that incorporate hygroscopic materials.

Optical sensors were developed as a response to the flaws of electrically based sensors. Electrical sensors, such as resistive sensors, require a current flow to the sensitized region. MOSFETs are susceptible to electromagnetic interferences that affect the sensing operation. Hysteresis error can occur, affecting the readings of sensors. Most electrical sensors require several nanoscale layers that act as electrodes and sensing layers. These nanoscale layers of functional electrical components require precise deposition to be able to sense stimuli 
Table 3

Different fibers/materials used in fiber sensors.

\begin{tabular}{lcccc}
\hline Material & Fiber & $\begin{array}{c}\text { Detection range } \\
(\% \mathrm{RH})\end{array}$ & Linearity/Sensitivity & Reference \\
\hline Zinc oxide & Plastic fiber & $50-70$ & $97.61 \%$ & $(35)$ \\
\hline 3-Aminopropyltriethoxysilane & Tapered FBG & $55-80$ & $2.51 \mathrm{pm} / \% \mathrm{RH}$ & $(16)$ \\
\hline Polyvinyl alcohol (PVA) & FBG & $40-75$ & - & $(36)$ \\
\hline GO & $\begin{array}{c}\text { Long-period } \\
\text { grating (LPG) }\end{array}$ & $40-100$ & $94.34 \%$ & $(37)$ \\
\hline Polyimide & Tilted FBG & $30-80$ & $0.0185 \mathrm{~nm} / \% \mathrm{RH}$ & $(38)$ \\
\hline No material & $\begin{array}{c}\text { Multi-mode } \\
\text { fiber }\end{array}$ & $20-100$ & $0.164 \mathrm{~nm} / \% \mathrm{RH}$ & $(39)$ \\
\hline
\end{tabular}

accurately. This raises the production cost of sensors. Electrical sensors are also less robust and most are not available in nanoscale form. This makes it difficult to embed such sensors in small and discrete structures. ${ }^{(40)}$

\section{Conclusions}

Hygroscopic characterization has usually not been reported in detail or been a focus of most studies, which have generally focused on structure morphologies, chemical structures, and electrical properties. It is important to note that for sensing that involves the detection of water molecules or environmental humidity, a hygroscopic study of the sensing material is necessary. A better understanding of hygroscopic behavior in materials may aid material synthesis and the improvement of the nanostructure. Every material possesses different forms of nanostructure with different capabilities of water uptake. The techniques discussed in this study are among the established methods of hygroscopic characterization. Hygroscopic materials are used in optical fibers. When materials are deformed upon water uptake, signals can be obtained and mapped from the material deformation. The ability of a material to take up water molecules and undergo a change in its physical form has led to the development of various sensors. Therefore, it is important for researchers to determine the best approach for characterizing a hygroscopic material.

\section{Acknowledgments}

This study was funded by the Ministry of Higher Education of Malaysia under the Fundamental Research Grant Scheme (FRGS), No. FRGS/1/2018/TK10/HWUM/02/2. 


\section{References}

1 Y. J. Wei, N. Silikas, Z. T. Zhang, and D. C. Watts: Dent. Mater. 27 (2011) 259. https://doi.org/10.1016/ j.dental.2010.10.015

2 D. M. Brewis, J. Comyn, A. K. Raval, and A. J. Kinloch: Int. J. Adhes. Adhes. 10 (1990) 247. https://doi. org/10.1016/0143-7496(90)90042-V

3 Q. Zheng, S. Liu, S. Yang, J. Lei, X. Peng, and C. Lin: 2016 IEEE Int. Conf. High Voltage Engineering and Application (ICHVE) (2016) 1. https://doi.org/10.1109/ICHVE.2016.7800746

4 J. G. Liang, E.-S. Kim, C. Wang, M.-Y. Cho, J.-M. Oh, and N.-Y. Kim: Sens. Actuators, B 265 (2018) 632. https://doi.org/https://doi.org/10.1016/j.snb.2018.03.093

5 R. J. Mortimer and R. J. Mayes: Sens. Actuators, B 128 (2007) 124. https://doi.org/10.1016/j.snb.2007.05.040

6 C. Y. Ho and Y.-S. Wu: Appl. Surf. Sci. 510 (2020) 145257. https://doi.org/https://doi.org/10.1016/ j.apsusc. 2020.145257

7 D. Han, L. Zhai, F. Gu, and Z. Wang: Sens. Actuators, B 262 (2018) 655. https://doi.org/10.1016/j.snb.2018.02.052

8 A. Sun, L. Huang, and Y. Li: Sens. Actuators, B 139 (2009) 543. https://doi.org/10.1016/j.snb.2009.03.064

9 Y. Ding, H. Hou, Y. Zhao, Z. Zhu, and H. Fong: Prog. Polym. Sci. 61 (2016) 67. https://doi.org/10.1016/ j.progpolymsci.2016.06.006

10 A. G. Tereshchenko: J. Pharm. Sci. 104 (2015) 3639. https://doi.org/10.1002/jps.24589

11 C. W. Harmon, R. L. Grimm, T. M. McIntire, M. D. Peterson, B. Njegic, V. M. Angel, A. Alshawa, J. S. Underwood, D. J. Tobias, R. B. Gerber, M. S. Gordon, J. C. Hemminger, and S. A. Nizkorodov: J. Phys. Chem. B 114 (2010) 2435. https://doi.org/10.1021/jp909661q

12 Y. Komazaki and S. Uemura: Sens. Actuators, B 297 (2019) 126711. https://doi.org/10.1016/j.snb.2019.126711

13 S. Lee and J.-W. Park: Sens. Actuators, B 298 (2019) 126883. https://doi.org/10.1016/j.snb.2019.126883

14 S. Shaziman, A. S. Ismail, M. H. Mamat, and A. S. Zoolfakar: IOP Conf. Series: Mater. Sci. Eng. 99 (2015) 012016. https://doi.org/10.1088/1757-899x/99/1/012016

15 A. Kołodziejczak-Radzimska and T. Jesionowski: Materials 7 (2014) 2833. https://doi.org/10.3390/ma7042833

16 A. M. Aris: J. Telecommun. Electron. Comput. Eng. (JTEC) 9 (2017) 1.

17 O. Singh, N. Kohli, and R. C. Singh: Sens. Actuators, B 178 (2013) 149. https://doi.org/10.1016/j.snb.2012.12.053

18 G. N. Narayanan and K. Annamalai: J. Mater. Sci.-Mater. Electron. 27 (2016) 12209. https://doi.org/10.1007/ s10854-016-5376-6

19 L. Coelho, D. Viegas, J. L. Santos, and J. M. M. M. de Almeida: Sens. Actuators, B 223 (2016) 45. https://doi. org/10.1016/j.snb.2015.09.061

20 R. Wahab, I. H. Hwang, H.-S. Shin, Y.-S. Kim, J. Musarrat, A. A. Al-Khedhairy, and M. A. Siddiqui: Intell. Nanomater. (2012) 183. https://doi.org/10.1002/9781118311974.ch5

21 B. P. Gangwar, S. C. Maiti, and S. Sharma: J. Solid State Chem. 256 (2017) 109. https://doi.org/10.1016/ j.jssc. 2017.08 .036

22 D. L. D. T. Babela and R. B. Lamorena: Chem. Pap. 74 (2020) 217. https://doi.org/10.1007/s11696-019-00870-2

23 M. Caponero, R. D'Amato, A. Polimadei, and G. Terranova: Measurement 125 (2018) 325. https://doi. org/10.1016/j.measurement.2018.04.072

24 H. Xie, G. Gong, Y. Wu, Y. Liu, and Y. Wang: Appl. Sci. 8 (2018) 430. https://doi.org/10.3390/app8030430

25 J. Wilkinson: Developing Graphene Oxide Membranes for the Purification of Water and Green Fuels. https:// www.azonano.com/article.aspx?ArticleID=4275 (June 23, 2020).

26 V. V. Perera, N. L. Fernando, B. Nissanka, and D. R. Jayasundara: Adsorption 25 (2019) 1543. https://doi. org/10.1007/s10450-019-00131-4

27 P. J. Thomas and J. O. Hellevang: Sens. Actuators, B 247 (2017) 284. https://doi.org/10.1016/j.snb.2017.02.027

28 M. Kim, S. G. Motti, R. Sorrentino, and A. Petrozza: Energy Environ. Sci. 11 (2018) 2609. https://doi. $\operatorname{org} / 10.1039 / \mathrm{C} 8 \mathrm{EE} 01101 \mathrm{~J}$

29 E. Hsiao, A. L. Barnette, L. C. Bradley, and S. H. Kim: ACS Appl. Mater. Interfaces 3 (2011) 4236. https://doi. org/10.1021/am200894h

30 K. Jaruwongrungsee, A. Tuantranont, Y. Wanna, A. Wisitsoraat, and T. Lomas: 2007 7th IEEE Conf. Nanotechnology (IEEE NANO) (2007) 316. https://doi.org/10.1109/NANO.2007.4601198

31 G. Sauerbrey: Z. Phy. 155 (1959) 206. https://doi.org/10.1007/BF01337937

32 R. P. Chiarello, J. Krim, and C. Thompson: Surf. Sci. 306 (1994) 359. https://doi.org/10.1016/00396028(94)90077-9

33 E. Stellrecht, H. Bongtae, and M. G. Pecht: IEEE Trans. Compon. Packag. Technol. 27 (2004) 499. https://doi. org/10.1109/TCAPT.2004.831777 
34 B. Han, D. Post, and P. Ifju: J. Strain Anal. Eng. Des. 36 (2001) 101. https://doi.org/10.1243/0309324011512568

35 Z. Harith, N. Irawati, M. Batumalay, H. A. Rafaie, G. Ii, S. W. Harun, R. Md Nor, and H. Ahmad: Indian J. Sci. Technol. 8 (2015) 1. https://doi.org/10.17485/ijst/2015/v8i35/85348

36 B. Rente, M. Fabian, Y. Chen, L. Vorreiter, H. Bustamante, T. Sun, and K. T. V. Grattan: IEEE Sens. J. 20 (2019) 1. https://doi.org/10.1109/JSEN.2019.2956826

37 Y. Qi, C. Jia, L. Tang, X. Zhang, C. Gong, Y. Liu, and X. Liu: Optik 188 (2019) 19. https://doi.org/10.1016/ j.ijleo.2019.05.043

38 B. Jiang, Z. Bi, Z. Hao, Q. Yuan, D. Feng, K. Zhou, L. Zhang, X. Gan, and J. Zhao: Sens. Actuators, B 293 (2019) 336. https://doi.org/10.1016/j.snb.2019.05.024

39 Y. Zhao, Y. Yuan, W. Gan, and M. Yang: Sens. Actuators, A 281 (2018) 48. https://doi.org/10.1016/ j.sna.2018.08.044

40 S. F. H. Correia, P. Antunes, E. Pecoraro, P. P. Lima, H. Varum, L. D. Carlos, R. A. S. Ferreira, and P. S. André: Sensors 12 (2012) 8847. https://doi.org/10.3390/s120708847 